\title{
Evaluation of the Cooperative Education Based on BP Neural Network-an Empirical Research
}

\author{
Ya Tian ${ }^{1,}$ a , Peng Yang ${ }^{1, b}$ \\ ${ }^{1}$ Xingtai University, Xingtai, 054001, China \\ aEmail: tianya_04@126.com, ${ }^{\mathrm{b}}$ Email:ypeng68@126.com
}

\begin{abstract}
Keywords: BP neural network; cooperative Education; evaluation; empirical research
Abstract. We aim to verify the mathematics model based on BP neural network is suitable for the evaluation of the present situation of cooperative education. Through analyzing the Cooperative education present situation index of a university, we determine the samples. By using SPSS software, we make factor analysis and reliability analysis to determine the influence factors of cooperative education. We establish the BP neural network model, calculate the data, and finally, we find the evaluation model based on BP neural network is suitable for the evaluation the present situation of cooperative education.
\end{abstract}

\section{Introduction}

Cooperative Education came into being in 1906. The new mode of education gained supports from the universities, the business community and the government and had a rapid development. In 1950s or 1960s, the mode of education was introduced very quickly by some European countries, and became a global education model. In 1983, the foundation of the World Association for Cooperative education became a symbol of higher education reform and the trend of the world ${ }^{[1]}$.

We start our study from the analysis of the social demand for applied talents. And then we find the important factors which influence cooperative education at home and abroad, construct the system of cooperative education, combine the curriculum resources and regional economy and enterprise resource, make the questionnaire analysis and data collection, establish the neural network model, take XingTai University as an example, analyze the strategy selection, the outcomes and costs, the benefits and other aspects of the model of cooperative education.

We believe that the following variables may be the main factors involved, including policy guidance, labor and social security, the management and the curriculum development, training simulation, scientific research and development, education and training, business management, three party protocol, school enterprise interaction etc.. Based on a questionnaire survey, we analyze the data from the school (college) leads, the full-time teachers, government officials, enterprise staffs to understand the relationship between the cooperation education and the relevant elements.

We conducted an education and training satisfaction evaluation. And the results is divided into five levels: the importance of the measures (less important, important, more important, very important and most important), directionality (not the direction, uncertain direction, something like the direction, the sure direction, very clear direction), the implementation difficulty level (very easy, easy, not easy, difficult, very difficult), current status of the school (fully achieve, achieve, partly achieve, rarely achieve, not achieve). We assign them 1-5 respectively. Likert ranking method is used in our study. Some indexes of hierarchies are in accordance with the actual situation.

The other factors are assigned with full score 10 points, and are divided into 1 to 10 levels. The standard for judging the factors affect the measuring index system. ${ }^{\text {[2] }}$

\section{Application of the BP neural network in cooperative education evaluation.}

The basic idea in our study is: make the factors influenced cooperative education as the input vector and the evaluation of the cooperative education as the output vectors in the BP neural network. Design reasonable network structure and the training sample, input the training sample in the network 
to calculate until the system error of network model meets our requirements. Then we get the model for the cooperation education evaluation.

1) The establishment of the model

Through questionnaire survey, we obtain the information of each index for different career. We set up a three layers BP neural network for the evaluation model of cooperation education. The input layer is the influence factors of a school and the output layer is the evaluation result. The evaluation result is graded into 1 to 10 points. So the output layer is 10 neurons.

A. data normalized processing:

We use premnmx function to normalize the learning samples, and tramnnx function to normalize the test sample. Finally we use postmnmx function to anti-normalize the test results.

B. the optimal number of hidden layer nodes algorithm:

How to select the best number of hidden layer nodes is still a problem to be solved. If the number of hidden layer nodes is too little, the neural network convergence speed will be too slow, and not easy to be convergence. On the contrary, if the number of hidden layer nodes is too much, the neural network topological structure will be too complex, the amount of calculation will be too large, and the error is not necessarily the minimum. The ideas of the number algorithm of hidden layer nodes: Follow the formula

$$
n_{i}=\sqrt{n+m}+a
$$

Here $n_{i}$ is the best number of hidden layer nodes. $n$ is the number of the input layer neuron. $m$ is the number of the output layer neuron. $a$ is a number from zero to twenty. For each number of hidden layer nodes, we choose the same training samples to train BP network topology structure, using unified test samples, with the minimum error as the criterion. Finally we find out the optimal number of hidden layer nodes.

C. the training of the network:

We train the network by using the momentum method and the improved BP algorithm of learning rate self-adaption adjustment. The input signal and the desired output are all gave into the network. The network will adjust the network weights and threshold constantly in the learning process. When the error between the output value and the desired output is less than the target, the learning process is over. Through the experiment many times, we find that when the target error is 0.01 , the accuracy of the judgment is the highest. Here the error precision should not be too high, because the input and output of the sample set have mixed noise signals. At the start of the learning, the adjustment of the network weights is aimed at the actual signal. But as the learning goes on, the structure of the network weights has the characteristics of noise. So excessive learning will introduce noise signals.

2) The implementation of the model

Step1. Establish a network through newff function. Based on the input sample data, the network determines the number of neurons in output layer automatically. And the number of hidden layer neurons, the layer number of the hidden layer, the transformation function of the hidden layer and output layer and the training algorithm function are determined by the user.

Step2. Initialize the network through the init function. The newff function creates network objects and call init function at the same time.

Step3. Train the network through the traiulm function according to the input vector $\mathrm{P}$, target vector $\mathrm{T}$ and the training parameters set up in advance of the sample.

Step4. Simulate the network through the Sim function. According to the trained network, simulation was carried out on the test data ${ }^{[4][5] \text {. }}$

We take the data of a questionnaire from 45 experts to the BP neural network as the input data and the corresponding overall evaluation as the output data. The code is as follows:

$\%$ NEWFF-generate a new forward neural network.

\%TRAIN-train the BP neural network.

$\%$ SIM-simulate the BP neural network.

$\% \mathrm{P}$ for the input vector.

$\% \mathrm{~T}$ for the output vector. 


\section{An empirical analysis of the cooperation education status indicators of Xingtai University.}

\section{(1) Cooperation education questionnaire and data processing.}

We conducted a questionnaire survey on all aspects of the cooperative education in our school. Fifty-eight questionnaires are taken back and seven repeated questionnaires. After finishing the questionnaire, we use the statistical analysis software SPSS18.0 to analyze the importance, the reform trend, the cost and difficulty and our current state of the cooperative education. The analysis process mainly includes the project analysis, factor analysis, reliability analysis.

The project analysis: the purpose of the project analysis is assessment on the authenticity of topics. The critical ratio (CR value) of is each item calculated. The calculation method is to arrange all the scores of the subjects from high to low. The top 25\% to 33\% of the score is High Score Group and the bottom $25 \%$ to $33 \%$ of the score is Low Score Group. Calculate the Significance test of average scores difference of the two groups in each topic. (Most of such data analysis let the highest and lowest 27\%as the high and low group boundary.) Make t-test for each sample on the topic difference between two groups. Delete the topics which t-test results don't achieve significance.

Factor analysis: after the project analysis, we make factor analysis to examine the construct validity of the questionnaires. The construct validity refers to the degree of how questionnaire can measure the theory or quality. The purpose of factor analysis is to find out the potential structure of the scale, reduce the number of topics.

Reliability analysis: after the factor analysis, reliability analysis is required. Reliability is the reliability and stability of the questionnaire. Reliability analysis method is used by L.J.Cronbach. Cronbach's coefficient is the most commonly used to assess reliability in psychological and education test. Alpha is for reliability coefficient (Cronbach's coefficient). Generally, the higher the coefficient, the higher the reliability is. In exploratory research, reliability is acceptable when the coefficient reach 0.70 . High reliability is deemed when the coefficient between $0.70-0.98$. While low reliability is considered when the coefficient is lower than 0.35 , and the reliability must be rejected.

\section{(2) Optimize the cooperation education factor index.}

In the measure of cooperation education situation in our school, we use the overall evaluation from 45 experts as the evaluation index. The sample is teachers, school managements, enterprise staffs and government officials with a total of 45 people. Let the overall evaluation of these professionals as $\mathrm{X}$,

$\mathrm{X}=(\mathrm{x} 1, \mathrm{x} 2, \mathrm{x} 3, \ldots, \mathrm{x} 45)$

$$
=(2,7,8,8,5,6,7,7,8,8,2,8,7,8,9,6,7,2,6,8,5,9,6,7,4,6,5,7,7,5,7,7,7,6,9,5,5,6,7,3,2,7,6,7,6){ }^{\prime}
$$

Through project analysis, factor analysis, reliability analysis, we determine the factors affecting the cooperative education in our school. They are mainly five types of index: training, curriculum, quality and security, policy, management. See table 1 . Because the coefficient of educational systems is less than 0.7 , we exclude the index here.

\section{(3)Cooperation education factor index optimization.}

The convergence curve below verifies that the elastic BP algorithm is very suitable for this kind of recognition. After training 393 times, the error meet the requirements of 0.001 . At this time learning process of BP neural network is completed.

According to the steps above, we have carried on the simulation training to the rest of the data. $X=[6,6,6,8,6,6,4,6,6,6,6,8,6,6,6,4,10,6,6,4,6,4,6,6,4,6 ; 6,4,4,4,6,6,4,4,6,4,6,4,4,6,4,4,6,4,4,4,4,4,6,4$, $8,4]^{\prime}$.

\section{The result of network training is $\quad \mathrm{a} 2=6.9439 \quad 6.0046$}

The simulation results of 6.9439 and 6.0046 are similar to the actual performance of 7 and 6. (See fig. 1.) It shows that the BP neural network used to evaluate learning performance is feasible.

Cooperation education evaluation based on BP artificial neural network model reflects the nonlinear relation between the influence factors of the cooperative education and the comprehensive evaluation results. So the influence of subjective factors in the cooperative education evaluation is reduced. It is an effective method to solve the problem of cooperative education evaluation. The model applied to the cooperative education evaluation can obtain satisfactory results. 
Table 1 the index system affecting cooperation education of Xingtai University

\begin{tabular}{|c|c|}
\hline The firstly indicators & The secondary indicators \\
\hline \multirow{9}{*}{ Training } & 14. Curriculum flexibility \\
\hline & 15. Scientific research \\
\hline & 19. Practice proportion \\
\hline & 23. Teachers background \\
\hline & 26. Training scenarios \\
\hline & 27. Simulating the working environment \\
\hline & 29. Education normalization \\
\hline & 4. School organization \\
\hline & 9. Curriculum development orientation \\
\hline \multirow{6}{*}{ Curriculum } & 10. The relationship between teachers and students \\
\hline & 12. Teaching content development \\
\hline & 13. Curriculum flexibility \\
\hline & 16. Combination of training and education \\
\hline & 25. Teachers' interaction between school and enterprise \\
\hline & 30. Teaching evaluation \\
\hline \multirow{4}{*}{ Quality and security } & 21. Combination of training and education \\
\hline & 23. Teachers background \\
\hline & 3. Mode of operation \\
\hline & 5. Course certification \\
\hline \multirow{4}{*}{ Policy } & 6. The labor security \\
\hline & 7. The three parties agreement \\
\hline & 24. The teacher hiring \\
\hline & 1. policy \\
\hline \multirow{2}{*}{ Management } & 2. Education management \\
\hline & 11.Interaction between practice and theory \\
\hline
\end{tabular}
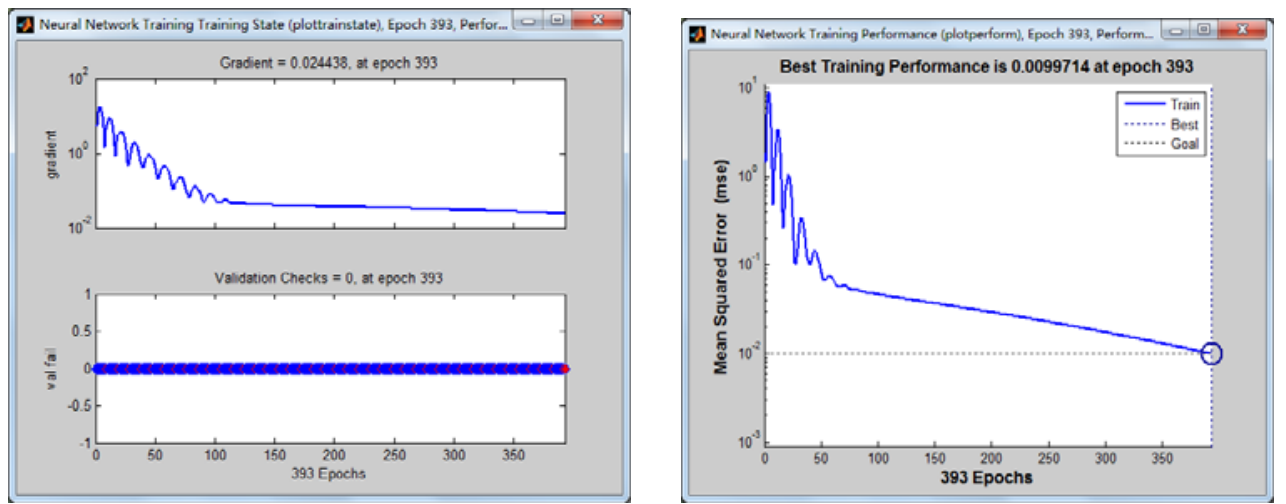

Fig. 1: Data training is completed, going into the simulation

\section{References}

[1]Jiefang Chen. Cooperative education theory and its practice in China[D].Huadong Normal University.2002. [2]Defeng Zhang. MATLAB neural network application[M]. Beijing: Mechanical industry press .2009.01.

[3]Minglong Wu. ,SPSS statistical applications[M]. Beijing: Railway press of China. 2000.09.

[4]MianMian Cai. Research on teaching quality evaluation in the college based on BP neural network[D].Hangzhou: Zhejiang University of Technology. 2009.

[5]Wang Xuhui, Huang Feihua. The Model of Teaching Quality Evaluation Based on BP Neural Networks and Its Application[J]. Research in Higher Education of Engineering. 2007.09. 